\title{
Update on Molecular Psychiatry: new publication guidelines and new ways to stay current
}

\begin{abstract}
Molecular Psychiatry (2009) 14, 463-464;
doi:10.1038/mp.2009.30
\end{abstract}

The field of molecular psychiatry has evolved considerably from when Molecular Psychiatry was launched in 1996. We present here brief and updated guidelines for publication as well as details on two innovative electronic resources-our blog and twitter.

We now receive over 700 papers annually and can only publish a very small fraction of what we receive. In the light of the resulting high-rejection rate, I am often asked what type of paper has the highest likelihood of acceptance. Here are some guidelines on the content and format that can lead to the highest likelihood of success.

On the important point of content, we seek work that is either conceptually novel or persuasively conclusive and that advances the field. Given the growth of approaches in psychiatric research, we are very open to the most diverse range of papers: from the epidemiological all the way to the most fundamental basic science, including in that broad range all aspects of translational and clinical research, pharmacology, genetics, genomics, proteomics, metabolomics, imaging, behavior and animal-model research. I cannot think of an innovative, groundbreaking or conclusive paper relevant to psychiatry that we would not be interested in. What is important is not so much the area, but either innovation and conceptual novelty or a definitive study that provides conclusive evidence.

As far as format is concerned, brevity is encouraged because of the tremendous pressure on our space. Most scientific work can benefit from a careful format and style revision aimed at achieving maximum clarity and brevity. Although your own story may be captivating to you, it is unlikely that reviewers or readers will have the time to go through lengthy details or speculation. Please build your paper around data and provide a succinct description of what led you to collect those data, including the guiding hypotheses and methods used, and then provide a brief discussion of your results in the context of where that particular area of research is now and where it is going. We truly look forward to receiving exciting work. Having introduced rapid review and turnaround to the publication process in psychiatry, we are fully committed to rigorous and very rapid peer review and electronic publication of the best work in the field.

One of our goals has been to foster dialogue in the field. Originally, that occurred in our Letters to the
Editor and News \& Commentary sections. However, the competition for our space makes the fostering of such dialogue increasingly difficult, as it is hard to justify rejecting a highly meritorious paper for lack of space as we dedicate space to interesting, but not critical, correspondence. Furthermore, there is now an increasing discrepancy between the immediacy of electronic publishing and the time constraints of print. In order to facilitate a dialogue in the field and to rapidly alert authors to emerging articles, I am personally creating two new resources for the field that will be mostly related to Molecular Psychiatry, but will occasionally include updates, news and comments on other material that is of relevance to our field. Those resources are a new Molecular Psychiatry blog and a twitter.

The Molecular Psychiatry blog can be found at http://molecularpsychiatry.blogspot.com. In this blog, we will give authors the option to provide summaries of their papers with links to the journal site containing the full article. Those summaries would appear in the blog in conjunction with Advance Online Publication. A dialogue around the paper and its subject matter can then rapidly develop in real-time with comments, questions and answers from the scientific community at large. Authors are not required to respond to comments, but may do so if they feel appropriate. As a safety measure, I will moderate this blog; therefore, material will only be posted with my approval. This will protect authors from inappropriate comments or nuisance. Having grown up in a military dictatorship that enforced official censorship of the press, I have a heartfelt commitment to freedom of speech and will only screen out truly inappropriate blog submissions. Authors and the public can e-mail me if they think that my screening is inappropriate. It is hoped that the blog will foster a lively real-time dialogue that will energize authors and readers.

Our second outreach innovation will be through twitter. For those interested in twittering on Molecular Psychiatry, just go into your twitter account and search for 'Julio Licinio.' Subscribe to my open twitter and as any exciting development emerges in Molecular Psychiatry or in other publications, I will twitter about those. For my older colleagues who do not know what twitter is, please get a tutorial from your children or young relatives, students, or anyone under the age of 30 years. You can also go to http:// www.twitter.com. Briefly, by subscribing to twitter you can tell others or hear from others what is 
happening now. I am using my twitter to rapidly disseminate exciting emerging data with relevant weblinks. The beauty of twitter is that each message can have a maximum of 140 characters. Those brief notifications are sufficient to keep you in touch with the latest findings, without the unnecessary distraction that would be caused by lengthy texts. I will mostly twitter take-home summaries of interesting papers as soon as they come out in Molecular Psychiatry online (ahead of print). Occasional twitters of groundbreaking work in the field, published elsewhere, will also be sent out-in moderation-as alerts of critical new data, without overwhelming your mailboxes.

J Licinio, Editor Miller School of Medicine, University of Miami, Miami, FL, USA E-mail: JLicinio@med.miami.edu 